CONCISE REPORT

\title{
Diagnostic characteristics of a gelatin based Waaler-Rose assay (Serodia-RA) for the detection of rheumatoid factor
}

\section{T Spiritus, P Verschueren, R Westhovens, X Bossuyt}

Ann Rheum Dis 2004;63:1169-1171. doi: 10.1136/ard.2003.013854

\begin{abstract}
Objective: To evaluate the diagnostic usefulness of a gelatin based Waaler Rose assay (Serodia-RA ${ }^{\circledR}$ ) for the detection of rheumatoid factor (RF) in rheumatoid arthritis (RA).

Methods: RF was measured by Serodia-RA and rate nephelometry in 90 patients with RA according to the revised ACR criteria and 102 patients with rheumatological diseases other than RA. Sensitivity, specificity, accuracy, likelihood ratios, and area under the curve using receiver operating characteristics (ROC) analysis were determined for both tests. Agreement between assays was assessed on 1657 consecutive samples.

Results: At equal specificity, Serodia-RA tended to be more sensitive than rate nephelometry $(0.66 \vee 0.58 ; p=0.04)$. ROC plots showed an area under the curve of 0.843 for Serodia-RA and 0.784 for nephelometry, providing further evidence that Serodia-RA was slightly better at differentiating between RA and non-RA arthropathy. Good agreement was found between both assays.

Conclusion: Serodia-RA is slightly more accurate than rate nephelometry for the detection of RF in RA, and a combination of both assays only marginally improves the diagnostic usefulness of RF detection. Use of two tests for detection of RF is not recommended. One test for detection of RF together with a more specific test, such as antibodies to cyclic citrullinated peptide, is suggested.
\end{abstract}

$\mathrm{R}$ heumatoid factor (RF) can be detected in the serum of the majority of patients with rheumatoid arthritis (RA) and is one of the seven classification criteria for RA proposed by the American College of Rheumatology (ACR). In patients with RA, the RF titre generally correlates with disease severity and predicts poor prognosis. ${ }^{2-4}$

Since the discovery of RF by Waaler in 1937, indirect haemagglutination has been a standard test for detection and quantification of RF. Alternative methods, like latex agglutination, enzyme linked immunosorbent assay (ELISA), and nephelometry, have since been developed for use in the routine clinical laboratory.

The original Waaler-Rose or sheep cell agglutination test detects RF through its ability to agglutinate sheep red blood cells sensitised with rabbit IgG. The Waaler-Rose assay has been described as less sensitive than nephelometry and latex agglutination for the detection of RF in patients with RA. ${ }^{5}$

No data are available, however, on the specificity and sensitivity of a modification of the Waaler-Rose, in which the red blood cells are replaced by artificial gelatin particles (Serodia-RA; Fujirebio Inc, Japan). Gelatin particles have no solid antigenic determinants, are biologically inactive, and incur minimal physical absorption of serum constituents, thus eliminating non-specificity derived from the erythrocyte carrier. We evaluated the performance of the
Serodia-RA for the detection of RF by comparing it with rate nephelometry.

\section{PATIENTS AND METHODS \\ Patients}

RF was measured by rate nephelometry and Serodia-RA in 90 patients with RA according to the 1987 ACR revised criteria ${ }^{1}$ (including 50 consecutive patients with "established" RA and 40 patients with early RA with arthritis onset $<1$ year) and 102 consecutive patients with rheumatological diseases other than RA admitted to the rheumatology department of our hospital between July and September 2002. The group of patients with diseases other than RA included 26 patients with osteoarthritis, 16 with spondyloarthropathy, 12 with psoriatic arthritis, 5 with unspecified non-inflammatory arthralgia, 5 with low back pain, 4 with polymyalgia rheumatica, 4 with systemic lupus erythematosus, 4 with crystal arthropathy, 3 with fibromyalgia syndrome, 3 with scleroderma, 2 with tendinitis, 2 with primary Raynaud's syndrome, 2 with spinal canal stenosis, 2 with post-traumatic rheumatic disease, 2 with rotator cuff impingement syndrome, 2 with carpal tunnel syndrome, 1 with mixed connective tissue disease, 1 with drug induced lupus, 1 with polymyositis, l with Wegener's disease, l with motor polyneuropathy, 1 with acrocyanosis, 1 with joint hyperlaxity, and 1 with unspecified systemic disease. None of these patients were suspected for RA at 1 year follow up. The two groups had a similar proportion of women $(59 \%$ and $66 \%$, respectively) and a similar median age (55 and 47 years, respectively). Additionally, RF was measured by both tests in 1657 consecutive serum samples submitted to the laboratory for determination of RF. These samples were collected from patients admitted to the University Hospitals Leuven between May 2001 and January 2002.

\section{Laboratory methods}

All sera were heat inactivated $\left(56^{\circ} \mathrm{C}, 30\right.$ minutes).

The Serodia-RA was performed according to the manufacturer's instructions. The titre was defined as the inverse number of the last final dilution giving agglutination, and a sample was considered positive if the titre was 40 or higher.

Rate nephelometry was performed on an Immage nephelometer (Beckman Instruments, California, USA) with Beckman reagents as described by the manufacturer's technical brochure. Results are given as IU/ml, and a result equal to or higher than 20 was considered positive.

Each of these tests was performed and evaluated by experienced technologists who were unaware of the other results and the patients' clinical data.

Abbreviations: ACR, American College of Rheumatology; RA, rheumatoid arthritis; RF, rheumatoid factor; ROC, receiver operating characteristics 
Table 1 Rheumatoid factor (RF) results of Serodia-RA and nephelometry in 90 patients with RA (including 40 patients with early RA) and in 102 disease controls - the number of patients with positive test results at two cut off values

\begin{tabular}{|c|c|c|c|c|}
\hline & \multicolumn{2}{|c|}{$\begin{array}{l}\text { Serodia-RA } \\
\text { (cut off value) }\end{array}$} & \multicolumn{2}{|c|}{$\begin{array}{l}\text { Nephelometry } \\
\text { (cut off value) }\end{array}$} \\
\hline & $\geqslant 40$ & $\geqslant 80$ & $\begin{array}{l}\geqslant 20 \\
\mathrm{IU} / \mathrm{ml}\end{array}$ & $\begin{array}{l}\geqslant 30 \\
\mathrm{IU} / \mathrm{ml}\end{array}$ \\
\hline $\begin{array}{l}\text { Patients with early RA } \\
(n=40) \\
\text { Patients with RA }(n=50) \\
\text { Disease controls }(n=102)\end{array}$ & $\begin{array}{l}30 \\
39 \\
12\end{array}$ & $\begin{array}{r}28 \\
31 \\
7\end{array}$ & $\begin{array}{r}26 \\
31 \\
9\end{array}$ & $\begin{array}{r}26 \\
26 \\
7\end{array}$ \\
\hline
\end{tabular}

\section{Statistical analysis}

The sensitivity and specificity of Serodia-RA and nephelometry at two different cut off levels were computed, together with the $95 \%$ confidence interval. McNemar's $\chi^{2}$ test for paired proportions was used to compare sensitivities of the tests. Additionally, accuracy (= sensitivity + specificity/2) and likelihood ratios were calculated. Areas under the receiver operating characteristics (ROC) curves with 95\% confidence intervals and significance of the difference were estimated using non-parametric ROC analysis. Significance was accepted at a p value of $<0.05$. Agreement between both tests was assessed using the $\kappa$ statistic on dichotomised data.

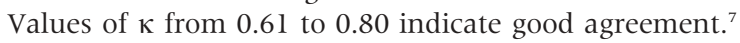

Data analysis was undertaken using Analyse-it, version 1.62, for Microsoft Excel.

\section{RESULTS}

The diagnostic performance of a gelatin based Waaler-Rose assay (Serodia-RA) for the detection of RF in RA was evaluated by comparing it with the performance of rate nephelometry. Tables 1 and 2 show the results obtained for 90 patients with clinically well defined RA and 102 patients with non-RA arthropathy. Although both tests had good performance characteristics, Serodia-RA tended to be more sensitive $(0.66 v 0.58 ; \mathrm{p}=0.04)$ than rate nephelometry at equal specificity $(0.93)$. As one would expect, at higher cut off values the sensitivity of both assays decreased while the specificity and positive likelihood ratio increased.

ROC analysis provided further evidence that Serodia-RA was slightly better at differentiating between RA and non-RA arthropathy than nephelometry with a significant difference $(\mathrm{p}<0.02)$ in the area under the curve: 0.843 (95\% CI 0.783 to 0.903 ) for Serodia-RA $v 0.784$ (95\% CI 0.716 to 0.853 ) for rate nephelometry (fig 1).

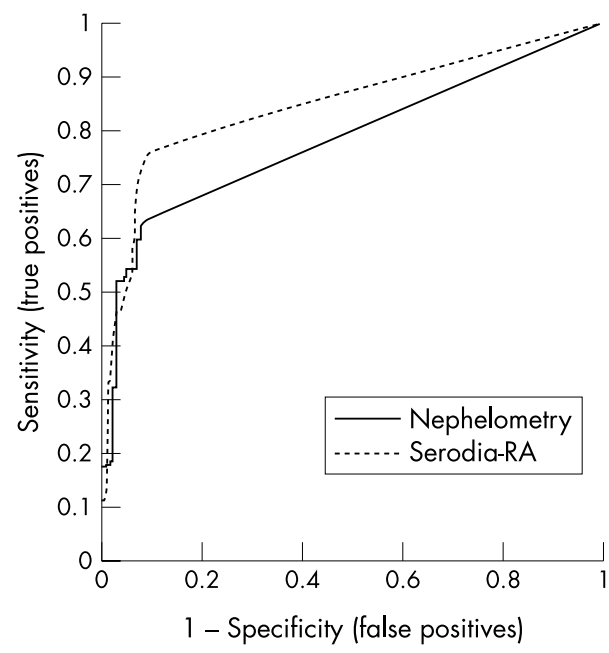

Figure 1 ROC curves for measurement of rheumatoid factor by Serodia-RA and rate nephelometry. Area under the curve for Serodia$\mathrm{RA}$ is 0.843 , for nephelometry 0.784 .

Table 3 Rheumatoid factor (RF) results measured by Serodia-RA and by nephelometry in 1657 consecutive samples submitted to the laboratory

\begin{tabular}{llc}
\hline \multirow{3}{*}{ Nephelometry } & \multicolumn{2}{l}{ Serodia-RA } \\
\cline { 2 - 3 } \cline { 2 - 2 } Positive $(\geqslant 20 \mathrm{IU} / \mathrm{ml})$, No $(\%)$ & $242(14.6)$ & Negative $(<40)$ \\
\hline Negative $(<20 \mathrm{IU} / \mathrm{ml})$, No $(\%)$ & $112(6.8)$ & $1277(77.1)$ \\
\hline
\end{tabular}

To assess agreement between both assays, RF results of 1657 consecutive samples measured by both assays were analysed. For 1519 samples there was agreement between the RF results: 242 samples were positive and 1277 samples negative by both methods. The $\kappa$ statistic was 0.73 and indicated good agreement between both methods. However, 138 discordant RF results were found: 112 samples were positive with Serodia-RA and negative with nephelometry, whereas only 26 samples were positive with nephelometry and negative with Serodia-RA (table 3). Medical records of these patients with discordant results were reviewed. Of the 112 Serodia-RA positive, nephelometry negative samples, 30 were from patients with RA and one from a patient with primary Sjögren's syndrome. None of the 26 Serodia-RA negative, nephelometry positive samples were from patients

Table 2 Rheumatoid factor (RF) results of Serodia-RA and nephelometry in 90 patients with RA (including 40 patients with early RA) and in 102 disease controls - sensitivity, specificity, accuracy, positive likelihood ratio $(L R+)$, and negative likelihood ratio $(L R-)$ of both tests, alone and combined

\begin{tabular}{llllll}
\hline & $\begin{array}{l}\text { Sensitivity } \\
(95 \% \mathrm{Cl})\end{array}$ & $\begin{array}{l}\text { Specificity } \\
(95 \% \mathrm{Cl})\end{array}$ & LR+ & LR- & Accuracy \\
\hline Serodia-RA $\geqslant 40$ & $0.77(0.67$ to 0.85$)$ & $0.88(0.80$ to 0.94$)$ & 6.4 & 0.3 & 0.82 \\
Serodia-RA $\geqslant 80$ & $0.66(0.55$ to 0.75$)$ & $0.93(0.86$ to 0.97$)$ & 9.4 & 0.4 & 0.79 \\
Nephelometry $\geqslant 20 \mathrm{IU} / \mathrm{ml}$ & $0.63(0.52$ to 0.73$)$ & $0.91(0.84$ to 0.96$)$ & 7.0 & 0.4 & 0.77 \\
Nephelometry $\geqslant 30 \mathrm{IU} / \mathrm{ml}$ & $0.58(0.47$ to 0.68$)$ & $0.93(0.86$ to 0.97$)$ & 8.3 & 0.5 & 0.75 \\
Serodia-RA $\geqslant 40 \mathrm{and}$ & $0.63(0.52$ to 0.73$)$ & $0.94(0.88$ to 0.98$)$ & 10.5 & 0.4 & 0.79 \\
nephelometry $\geqslant 20 \mathrm{IU} / \mathrm{ml}$ & $0.77(0.67$ to 0.85$)$ & $0.85(0.77$ to 0.92$)$ & 5.1 & 0.3 & 0.81 \\
$\begin{array}{l}\text { Serodia-RA } \geqslant 40 \text { or } \\
\text { nephelometry } \geqslant 20 \mathrm{IU} / \mathrm{ml}\end{array}$ & & & & & \\
\hline
\end{tabular}


with RA and one was from a patient with primary Sjögren's syndrome.

With Serodia-RA more positive RF results were found than with nephelometry $(21.4 \%$ and $16.2 \%$, respectively; table 3$)$. These results further suggested higher sensitivity but lower specificity for Serodia-RA than with nephelometry at the chosen cut off values.

\section{DISCUSSION}

We evaluated the diagnostic usefulness of Serodia-RA for the detection of RF in RA. RF was measured by Serodia-RA and by rate nephelometry in samples taken from patients with RA and from patients with rheumatological diseases other than RA. Our data showed slightly better performance characteristics for Serodia-RA. Good agreement was found between both assays in consecutive samples. A combination of both assays only marginally improved the diagnostic usefulness of RF detection compared with Serodia-RA or nephelometry alone, with a slightly higher specificity and positive likelihood ratio if both assays were positive (table 2). The diagnostic characteristics of RF tests are in part dependent on the characteristics of the clinical population in which the test is used. The test characteristics found in the present study will therefore not necessarily be in accordance with those seen in other clinic populations. However, the ratio of the diagnostic qualities of the different RF tests is not affected by these population characteristics, so that the Serodia-RA can be accepted as being slightly better than rate nephelometry.

On the other hand, there are several advantages in using nephelometric techniques in measuring RF; it is fast, truly quantitative, and has a good precision. The total cost of the test depends on several factors, including reagents, materials, and technician's time. For example, in our laboratory, the cost of materials and reagents (including controls and general laboratory costs) was calculated to be $€ 2.42$ for $R F$ measured by nephelometry and $€ 3.03$ for RF measured by Serodia-RA. The cost for technician/operator was $€ 3.82$ for nephelometry (offered five times a week) and $€ 4.71$ for the Serodia-RA (offered twice a week). If the Serodia-RA test was offered five times a week, then the operator's cost would increase to about $€ 11$. However, for a smaller laboratory where RF tests are performed less frequently the Serodia-RA is not necessarily more expensive than nephelometry.

For historical and technical reasons several clinical laboratories provide two tests for the detection of RF as part of their test portfolio. We do not recommend this practice for two reasons. Firstly, our results show that performing two tests for the detection of RF only marginally improves diagnostic usefulness compared with one test. Secondly, other autoantibody-antigen systems such as antibodies to citrulline containing peptides have been reported to be more specific than RF for the diagnosis of RA. Several publications have recommended a combination of RF and a more specific test (for example, antibodies to cyclic citrullinated peptide) for the diagnosis of RA. . $^{8-10}$

Therefore we think that each laboratory using two tests for RF should select just one, judging on diagnostic characteristics, cost price, and practicability, and complement it with a more specific test. In our hospital we have decided not to perform the Serodia-RA any longer, despite the fact that it has slightly better diagnostic characteristics. The nephelometric assay is complemented with determination of antibodies to cyclic citrullinated peptide.

\section{Authors' affiliations}

T Spiritus, X Bossuyt, Department of Laboratory Medicine, University Hospitals Leuven, Belgium

P Verschueren, R Westhovens, Department of Rheumatology, University Hospitals Leuven, Belgium

Correspondence to: Professor X Bossuyt, Department of Laboratory Medicine, Immunology, University Hospitals Leuven, Herestraat 49, B-3000 Leuven, Belgium; xavier.bossuył@uz.kuleuven.ac.be

Accepted 17 October 2003

\section{REFERENCES}

1 Arnett FC, Edworthy SM, Bloch DA, McShane DJ, Fries JF, Cooper NS, et al. The American Rheumatism Association 1987 revised criteria for the classification of rheumatoid arthritis. Arthritis Rheum 1988;31:315-24.

2 Bukhari M, Lunt M, Harrison BJ, Scott DGI, Symmons DPM, Silman AJ. Rheumatoid factor is the major predictor of increasing severity of radiographic erosions in rheumatoid arthritis. Arthritis Rheum 2002;46:906-12.

3 Jansen LMA, van der Horst-Bruinsma IE, van Schaardenburg D, Bezemer PD, Dijkmans BAC. Predictors of radiographic joint damage in patients with early rheumatoid arthritis. Ann Rheum Dis 2001;60:924-7.

4 Drossaers-Bakker KW, Zwinderman AH, Vlieland TPM, Van Zeben D, Vos K, Breedveld FC, et al. Long-term outcome in rheumatoid arthritis: a simple algorithm of baseline parameters can predict radiographic damage, disability, and disease course at 12-year followup. Arthritis Rheum 2002;47:383-90.

5 Ulvestad E, Kanestrøm A, Madland TM, Thomassen E, Haga HJ. Clinical utility of diagnostic tests for rheumatoid factor. Scand J Rheumatol 2001;30:87-91.

6 Roberts-Thomson PJ, McEvoy R, Langhans T, Bradley J. Routine quantification of rheumatoid factor by rate nephelometry. Ann Rheum Dis 1985;44:379-83.

7 Altman DG. Practical statistics for medical research. London: Chapman and Hall, 1991:403-9.

8 Bizarro N, Mazzanti G, Tonutti E, Villalta D, Tozolli R. Diagnostic accuracy of the anti-citrulline antibody assay for rheumatoid arthritis. Clin Chem 2001;47:1089-93.

9 Lee DM, Schur PH. Clinical utility of the anti-CCP assay in patients with rheumatic diseases. Ann Rheum Dis 2003;62:870-4.

10 Kroot EJ, de Jong BA, van Leeuwen MA, Swinkels H, Van Den Hoogen FH, Van ' $t$ Hof $M$, et al. The prognostic value of anti-cyclic citrullinated peptide antibody in patients with recent-onset rheumatoid arthritis. Arthritis Rheum 2000:43:1831-5 